\title{
Bordetella bronchiseptica Pneumonia Post-Liver Resection in a Patient with Liver Cirrhosis and Cholangiocarcinoma
}

\author{
Hugo Bonatti, ${ }^{1,2}$ Brian Craig, Nithin Theckumparampil, John Tarpley, ${ }^{1}$ Nadja Colon, \\ Addison May, and Lee Gorden ${ }^{1}$
}

\begin{abstract}
Background: Although Bordetella bronchiseptica is primarily an animal pathogen, cases of human disease caused by this pathogen have been published recently, most frequently pneumonia in immunocompromised patients. In human disease, transmission through animal vectors may play a key role. Although no standardized sensitivity testing is available for this pathogen in human disease, animal isolates are sensitive to most $\beta$-lactam antibiotics. Case Report: A 62-year-old Caucasian male with Child-Pugh class A cirrhosis caused by chronic hepatitis C infection underwent uneventful left lateral segmentectomy for a $3 \mathrm{~cm}$ cholangiocarcinoma. Within $48 \mathrm{~h}$, he developed altered mental status, temperature of $39.4^{\circ} \mathrm{C}$, leukocytosis (white blood cell count: $13,000 / \mathrm{mm}^{3}$ ), and dyspnea followed by hypotension requiring vasopressor support and intubation. Computed tomography (CT) scan demonstrated left lower lobe pneumonia. Empiric antibiotic therapy including vancomycin $(1 \mathrm{~g}$ every $12 \mathrm{~h})$ and piperacillin-tazobactam $(3.5 \mathrm{~g}$ every $6 \mathrm{~h})$ was initiated and his signs of sepsis resolved within two days. Bordetella bronchiseptica was cultured from sputum. Upon questioning, the patient reported close contact with several pet cats on the days prior to admission. Antibiotics were continued for a total of seven days and he was discharged in good condition doing well at his six-month follow-up.

Conclusions: Immunocompromised patients may develop infection with Bordetella bronchiseptica especially if they are in close contact with animals known to be a reservoir of this pathogen. If diagnosed early and treated appropriately, the outcome is favorable.
\end{abstract}

$\boldsymbol{B}$ ORDETELLA BRONCHISEPTICA is a small, gram negative, rod-shaped bacterium of the genus Bordetella [1]. Rarely reported in human beings, it is a common cause of respiratory disease in farm, wild, and pet animals [2,3]. Cats, dogs, rabbits, pigs, cattle, polar bears, sloths, and rodents may harbor this organism [4-6]. Bordetella bronochiseptica is closely related to $B$. pertussis but does not express pertussis toxin and is not considered a primary human pathogen [1]. However, an increasing number of $B$. bronchoseptica human infections have been reported recently [7-9]. Immunocompromised patients are affected by the pathogen most commonly [10,11]. A large series of patients with cancer with $B$. bronchiseptica infections was recently published [12]. Pneumonia is the most common manifestation in human beings, however, reports on other infections such as one patient with liver cirrhosis and spontaneous bacterial peritonitis caused by B. bronchiseptica are available [9]. There is evidence that transmission through animal vectors plays a key role [7,8,13-16]. Register et al. [8] documented transmission of B. bronchiseptica from a cat to a patient with cystic fibrosis.

Pneumonia is one of the most common infections complicating major surgical procedures [17]. The most common pathogens isolated in this type of infection include Streptococcus pneumoniae, Haemophilus influenzae, Staphylococcus aureus, Klebsiella spp., and Pseudomonas aeruginosa with a number of additional pathogens to be considered [18]. The longer patients are hospitalized and ventilator dependent, the more likely they are to acquire hospital pathogens, many being multi-drug resistant [19]. Rare organisms including bacteria, viruses, and fungi may be found in patients with

\footnotetext{
${ }^{1}$ Department of Surgery, Vanderbilt University Medical Center, Nashville, Tennessee.

${ }^{2}$ University of Maryland Community Medical Group-Surgical Care, Easton, Maryland.
}

(c) Hugo Bonatti et al. 2016; Published by Mary Ann Liebert, Inc. This Open Access article is distributed under the terms of the Creative Commons License (http://creativecommons.org/licenses/by/4.0), which permits unrestricted use, distribution, and reproduction in any medium, provided the original work is properly credited. 
severe underlying diseases such as renal failure, hepatic failure, diabetes mellitus, and in particular, in severely immunocompromised individuals [18].

\section{Case Report}

A 62-year-old Caucasian male with Child-Pugh class A liver cirrhosis caused by chronic hepatitis $\mathrm{C}$ infection was diagnosed with a $3 \mathrm{~cm}$ lesion in the left lateral liver segments. On biopsy, cholangiocarcinoma was found. He had a history of type 2 diabetes mellitus, peptic ulcer disease, hypertension, depression, and asymptomatic $4 \mathrm{~cm}$ abdominal aortic aneurysm. He had no signs or symptoms of liver failure pre-operatively. The first two days after left lateral segmentectomy were uneventful with the exception of persistent altered mental status and disorientation. On the third post-operative day, the patient developed a fever (temperature, $39.4^{\circ} \mathrm{C}$ ), with oxygen saturation of $98 \%$ on three liters of oxygen via nasal cannula. The patient progressed to hypotension with his lowest non-invasive blood pressure of $80 / 45 \mathrm{~mm} \mathrm{Hg}$. Norepinephrine at a dose of up to $7 \mathrm{mcg} / \mathrm{kg}$ per hour was given and the patient's blood pressure normalized. His incision did not demonstrate any erythema, induration or drainage; his abdomen was non-tender and not distended. International normalized ratio (INR) and total bilirubin peaked at 1.3 and 2.0, respectively; his white blood cell (WBC) count was $13,000 / \mathrm{mm}^{3}$. Computed tomography (CT) demonstrated left lower lobe pneumonia (Fig. 1); there was no intra-abdominal fluid collection. Blood cultures and tracheal aspirates were obtained, followed by administration of vancomycin ( $1 \mathrm{~g}$ every $12 \mathrm{~h})$ and piperacillin-tazobactam $(3.5 \mathrm{~g}$ every $6 \mathrm{~h}$ ) empiric therapy. The fever subsided, norepinephrine was weaned within $24 \mathrm{~h}$, oxygenation improved, and oxygen was weaned. His mental status returned to normal over the next two days and the patient continued with an uneventful recovery. Blood cultures remained negative, however, $B$. bronchiseptica was cultured from sputum. Antibiotics were continued for a total of seven days. Upon questioning, the patient reported that he had had close contact with several pet cats on the days prior to surgery. He was discharged home in good condition and did well on his six-month follow-up with no signs of recurrent infection or tumor.

\section{Discussion}

We report a patient with cholangiocarcinoma and liver cirrhosis undergoing a major surgical procedure who developed pneumonia as a result of a rare human pathogen. Although his pets were not tested for carriage of the pathogen, we believe that they are the source of his infection. In the series by Yacoub et al. [12], no clear transmission from pets can be assumed. Redelmann-Sidid et al. [13] reported recently a patient with glioblastoma on temozolomide therapy with kitten-transmitted B. bronchoseptica infection. Wernli et al. [7] summarized recently eight cases of $B$. bronchoseptica infection in human beings, the majority of whom had severe underlying disease and three of whom had been exposed to cats. Our patient is only the eleventh reported patient with cancer and the first with malignant disease of the liver with $B$. bronchiseptica infection. We believe that our patient came to the hospital colonized with B. bronchiseptica after contact with his pet cats. He was not able to clear the pathogen because of his impaired immune function in the course of his liver cirrhosis and cancer. During intubation, the pathogen may have been introduced into his airways. For peri-operative prophylaxis the patient received a single dose of cefazolin, which did not control growth of the bacterium. Within $48 \mathrm{~h}$ he developed pneumonia with signs of hemodynamic instability. Our patient had a dense infiltrate, which corresponds to findings reported recently by Patel et al. [20] in two immunosuppressed patients. Piperacillin-tazobactam was used for empiric therapy in our patient similar to one patient in the series of Yacoub et al. [12] and our patient responded to this therapy. Of note, no clear guidelines are available currently for human infection, however, various agents including $\beta$-lactam $/ \beta$-lactamase inhibitor combinations, carbapenems, tetracycline, and fluouroquinolones have been used successfully. In the patient reported by Dlamini et al. [9] ciprofloxacin was used because intracellularly active agents may show better response. Animal isolates of B. bronchiseptica have

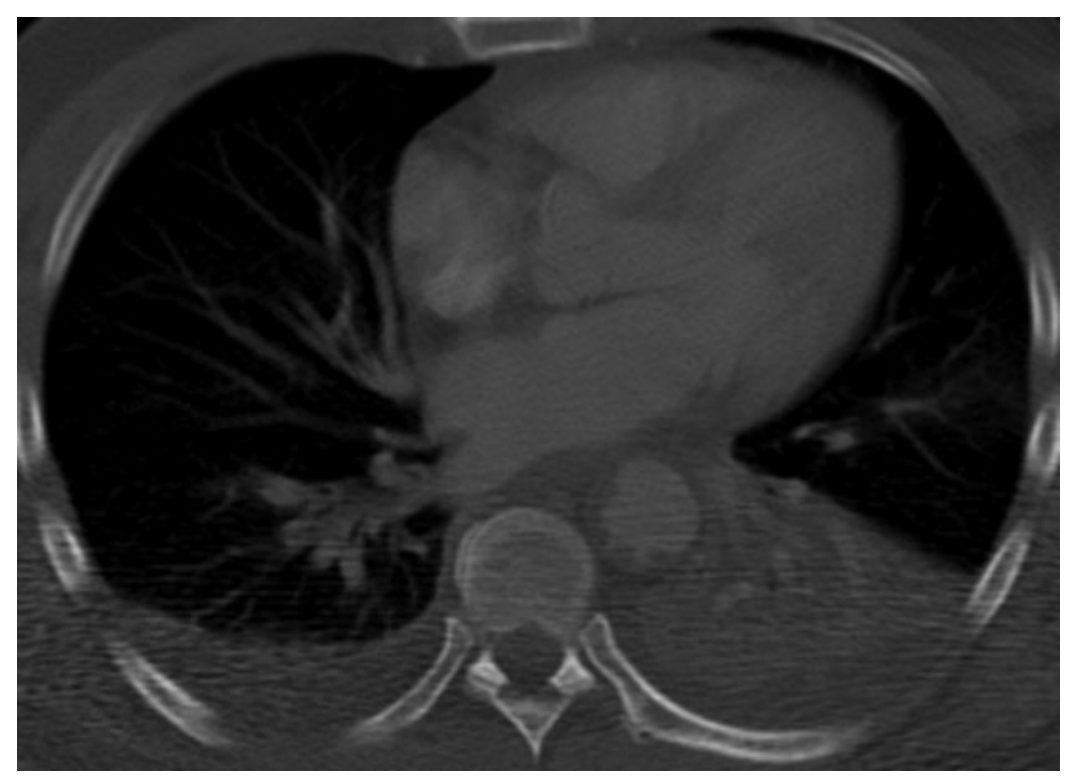

FIG. 1. Computed tomography scan shows dense left lower lobe infiltrate. 
been shown to be susceptible to a variety of agents including tetracycline, fluouroquinolones, and $\beta$-lactam antibiotics $[21,22]$. Trimethoprim as well as sulfonamides are considered ineffective. For animals, a nasal immunization is available [23] but this has not been done thus far in human beings.

Immunocompromised patients, including patients with cancer, may develop infection with $B$. bronchiseptica especially if they are in close contact with various animals known to be a reservoir of this pathogen [8,24]. If diagnosed early and treated appropriately, the outcome is favorable.

\section{Author Disclosure Statement}

No competing financial interests exist.

\section{References}

1. Goodnow RA. Biology of Bordetella bronchiseptica. Microbiol Rev 1980;44:722-738.

2. Gross R, Keidel K, Schmitt K. Resemblance and divergence: The "new" members of the genus Bordetella. Med Microbiol Immunol 2010;199:155-163.

3. Egberink H, Addie D, Belak S, et al. Bordetella bronchiseptica infection in cats. ABCD guidelines on prevention and management. J Feline Med Surg 2009;11610-614.

4. Lacasse C, Gamble KC. Tracheitis associated with Bordetella bronchiseptica in a polar bear (Ursus maritimus). $\mathrm{J}$ Zoo Wildl Med 2006;37:190-192.

5. Hammond EE, Sosa D, Beckerman R, Aguilar RF. Respiratory disease associated with Bordetella bronchiseptica in a Hoffmann's two-toed sloth (Choloepus hoffmanni). J Zoo Wildl Med 2009;40:369-372.

6. Zhao Z, Wang C, Xue Y, et al. The occurrence of Bordetella bronchiseptica in pigs with clinical respiratory disease. Vet J 2011;188:337-340.

7. Wernli D, Emonet S, Schrenzel J, Harbarth S. Evaluation of eight cases of confirmed Bordetella bronchiseptica infection and colonization over a 15-year period. Clin Microbiol Infect 2011;17:201-203.

8. Register KB, Sukumar N, Palavecino EL, et al. Bordetella bronchiseptica in a paediatric cystic fibrosis patient: Possible transmission from a household cat. Zoonoses Public Health 2012;59:246-250.

9. Dlamini NR, Bhamjee A, Levick P, et al. Spontaneous bacterial peritonitis and pneumonia caused by Bordetella bronchiseptica. J Infect Dev Ctries 2012;6:588-591.

10. El Khatib N, Ferroni A, Le Bourgeois M, et al. Persistent Bordetella bronchiseptica infection in a child with cystic fibrosis: Relationship to bacterial phenotype. J Cyst Fibros 2015;14:E13-15.

11. Washington MA, Agee WA, Kajiura L, et al. A case of Bordetella brochiseptica at a military medical facility in Hawai'i: Phenotypic and molecular testing of an uncommon human pathogen. Hawaii J Med Public Health 2015; 74:230-233.

12. Yacoub AT, Katayama M, Tran J, et al. Bordetella bronchiseptica in the immunosuppressed population-A case series and review. Mediterr J Hematol Infect Dis 2014; 6:e2014031.

13. Redelman-Sidi G, Grommes C, Papanicolaou G. Kittentransmitted Bordetella bronchiseptica infection in a patient receiving temozolomide for glioblastoma. J Neurooncol 2011;102:335-339.
14. Gisel JJ, Brumble LM, Johnson MM. Bordetella bronchiseptica pneumonia in a kidney-pancreas transplant patient after exposure to recently vaccinated dogs. Transpl Infect Dis 2010;12:73-76.

15. Mazumder SA, Cleveland KO. Bordetella bronchiseptica bacteremia in a patient with AIDS. South Med J 2010; 103:934-935.

16. Hadley K, Torres AM, Moran J, Schiller B. Bordetella bronchiseptica peritonitis-Beware of the dog! Perit Dial Int 2009;29:670-671.

17. Tejada Artigas A, Bello Dronda S, Chacon Valles E, et al. Risk factors for nosocomial pneumonia in critically ill trauma patients. Crit Care Med 2001;29:304-309.

18. Gastmeier P, Sohr D, Geffers C, et al. Early- and late-onset pneumonia: Is this still a useful classification? Antimicrob Agents Chemother 2009;53:2714-2718.

19. Bonatti HJ, Colon N, Ott M, et al. Trimethoprim-associated hyperkalemia in a young trauma victim. Surg Infect 2011; 12:419-420.

20. Patel AK, Prescott-Focht JA, et al. Imaging findings in human Bordetella bronchiseptica pneumonia. J Thorac Imaging 2011;26:W146-149.

21. Kadlec K, Kehrenberg C, Wallmann J, Schwarz S. Antimicrobial susceptibility of Bordetella bronchiseptica isolates from porcine respiratory tract infections. Antimicrob Agents Chemother 2004;48:4903-4906.

22. Zhao Z, Xue Y, Wang C, et al. Antimicrobial susceptibility of Bordetella bronchiseptica isolates from pigs with respiratory diseases on farms in China. J Vet Med Sci 2011;73:103-106.

23. Lehar C, Jayappa H, Erskine J, et al. Demonstration of 1year duration of immunity for attenuated Bordetella bronchiseptica vaccines in dogs. Vet Ther 2008;9:257-262.

24. Galeziok M, Roberts I, Passalacqua JA. Bordetella bronchiseptica pneumonia in a man with acquired immunodeficiency syndrome: A case report. J Med Case Rep 2009;3:76.

Address correspondence to: Dr. Hugo Bonatti University of Maryland Community Medical Group-Surgical Care 503 Dutchman's Lane Easton, MD 21601

E-mail: Hugo.bonatti@dr.com

$\begin{aligned} & \text { Abbreviations Used } \\ \mathrm{CT} & =\text { computed tomography } \\ \mathrm{INR}= & \text { international normalized ratio } \\ \mathrm{WBC}= & \text { white blood cell }\end{aligned}$

Cite this article as: Bonatti $\mathrm{H}$, Craig $\mathrm{B}$,

Theckumparampil N, Tarpley J, Colon N, May A, Gorden L (2016) Bordetella bronchiseptica pneumonia post-liver resection in a patient with liver cirrhosis and cholangiocarcinoma. Surgical Infections Case Reports 1:1, 112-114, DOI: 10.1089/crsi.2016.0026 